\title{
Treatment of Atopic Dermatitis with Biologic Drugs
}

\author{
Gabriella Fabbrocini - Maddalena Napolitano • Matteo Megna •
}

Nicola Balato $\cdot$ Cataldo Patruno

Received: July 19, 2018 / Published online: September 4, 2018

(C) The Author(s) 2018

\section{ABSTRACT}

Atopic dermatitis (AD) is a chronic, pruritic, inflammatory skin disease which predominately affects children and usually clears up during infancy or childhood. However, AD may persist with a chronic relapsing course until adulthood or develop at a later age. AD treatment can often be complicated. Treating moderate-to-severe $\mathrm{AD}$ can be challenging: only a few therapeutic options are available, with cyclosporine being the only approved and labeled systemic drug. In the last few years, advances in the knowledge of $\mathrm{AD}$ pathogenesis have been made that can provide the basis for developing new topical and systemic drugs. Among them, biologic drugs targeting specific cytokines involved in the development of the disease will probably revolutionize $\mathrm{AD}$ therapy. Currently, dupilumab, a monoclonal antibody that binds to the

Enhanced Digital Features To view enhanced digital features for this article go to https://doi.org/10.6084/ m9.figshare.6990209.

G. Fabbrocini $\cdot$ M. Megna $\cdot$ N. Balato

C. Patruno $(\square)$

Department of Dermatology, University of Naples

Federico II, Naples, Italy

e-mail: cataldopatruno@libero.it

M. Napolitano

Department of Medicine and Health Science

"Vincenzo Tiberio", University of Molise,

Campobasso, Italy shared alpha chain subunit of the receptors for IL-4 and IL-13, is the only biologic drug licensed for the treatment of $\mathrm{AD}$ in adults. However, other biologic drugs that selectively target some key cytokines in AD pathogenesis (IL-13, IL-31, and IL-22) are also being studied. In this review, we discuss all of the biologic drugs that have been studied for AD treatment.

Keywords: Atopic dermatitis; Biologic drug; Biologics; Therapy; Treatment

\section{INTRODUCTION}

Atopic dermatitis (AD) is the most common inflammatory skin disease of childhood, affecting about $20 \%$ of children [1]. AD usually resolves before adolescence. However, it sometimes persists in adulthood (persistent $\mathrm{AD}$ ), and in some cases the disease starts in adulthood (adult-onset $\mathrm{AD}$ ) [2]. It is reported that the prevalence of childhood AD is steadily increasing, especially in industrialized countries [3]. In the same way, it is likely that the frequency of the adult form has also been increasing in recent years, as even reported for elderly patients [4]. Current prevalence data regarding adult $\mathrm{AD}$ are very variable, with values ranging from 0.3 to $14.3 \%$, even though most authors agree that $\mathrm{AD}$ affects $1-3 \%$ of adults [5]. AD has a chronic relapsing course and significantly 
affects the quality of life of patients, especially adults with severe and long-lasting AD [6]. The diagnosis of adult $\mathrm{AD}$ is usually clinical, since traditional diagnostic criteria used for children are not always applicable to adults [5]. Studies are now underway that aim to better define the clinical characteristics of the disease $[7,8]$. In mild cases, topical treatment includes corticosteroids and calcineurin inhibitors in addition to emollient agents, which are the cornerstone of treatment in all cases of $\mathrm{AD}$ [1]. Adjunctive systemic treatment is needed in moderate-tosevere cases [9]. Phototherapy, cyclosporine A (CsA), methotrexate, and azathioprine are the main immunosuppressors used in $\mathrm{AD}$ treatment, while corticosteroids are used only for acute exacerbations [1]. Currently, cyclosporine is the only labeled medicament available for the treatment of AD. Due to the chronicity of the condition, long-term immunosuppressive treatment is often required. Subsequently, continuous follow-up is needed, as all of the abovementioned drugs may have important side effects [9]. Moreover, these therapies show different efficacies [9]. In recent years, several studies have elucidated the pathogenesis of the disease, especially as regards the roles of some cytokines [10]. This has led to the introduction of new drugs targeting individual cells or a mediator of the inflammatory response [1,9]. Among these new drugs, biologic agents are playing a leading role. Currently, the monoclonal antibody dupilumab is the only biologic drug licensed for the treatment of AD in adults $[1,7]$. Dupilumab blocks interleukin (IL)-4 and IL-13, two cytokines that play a key role in the development of type 2 helper (Th2) lymphocyte-mediated skin inflammation in $\mathrm{AD}$ [11]. Other biologic medicaments-drugs that selectively inhibit some cytokines (namely IL-13, IL31 , and IL-22) that play prominent roles in the pathogenesis of DA-are also under investigation [1].

This review summarizes currently available biologic therapies for adult $\mathrm{AD}$ based on an analysis of the literature in this field, and highlights possible future scenarios for this kind of treatment.

\section{METHODS}

For the current review, relevant literature published in English was searched for. The studies we selected concerned systemic biologic therapy of adult atopic dermatitis. We selected controlled phase II and III trials as well as reviews, guidelines, and consensus. Research was carried out by searching the following databases through to June 30, 2018: PubMed, Embase, The Cochrane Library, Google Scholar, EBSCO, and clinicaltrials.gov. The following keywords were used: "atopic dermatitis," "atopic eczema," "adult atopic dermatitis," "adult atopic eczema," "systemic treatment," "systemic therapy," "biologics," "monoclonal antibody," "IL-4 monoclonal antibody," "IL-13 monoclonal antibody," "IL-31 monoclonal antibody," and "IL-22 monoclonal antibody." This article is based on previously conducted studies and does not involve any new study of human or animal subjects performed by any of the authors.

\section{BIOLOGIC DRUGS}

The development of biological therapies has progressed rapidly in the last few years. Biologic drugs are a class of pharmacological agents that are engineered to target specific mediators of inflammation. Recently, multiple clinical trials have demonstrated the efficacy of targeted therapy involving the blocking of cytokines or mediators that play a pivotal role in the pathogenesis of AD. Dupilumab is currently the only biologic drug that has been approved by both the US Food and Drug Administration (FDA) and the European Medicines Agency (EMA) for the treatment of adult patients with inadequately controlled moderate-to-severe $\mathrm{AD}$ [12]. Biological drugs appear to be promising treatments for adult $\mathrm{AD}$ patients because they offer more convenient dose regimens and allow less frequent laboratory monitoring than other systemic therapies, as well as having fewer side effects [1]. Future studies are needed to fulfill unmet needs and achieve better standards of care for $\mathrm{AD}$ patients, to clarify the roles of other biologic drugs under investigation for $\mathrm{AD}$ 
therapy, and to completely revolutionize adult $\mathrm{AD}$ treatment.

\section{IL-4 and/or IL-13 Inhibitors}

Dupilumab is a fully human monoclonal antibody that binds to the shared alpha chain subunit of the receptors for IL-4 and IL-13 [13]. This results in downregulation of receptor signaling downstream of the JAK/STAT pathway, which regulates the expression of many genes involved in the pathogenesis of $\mathrm{AD}$ [14]. In March 2017, dupilumab received its first national approval, from the USA, for its use in the treatment of adult patients with moderateto-severe $\mathrm{AD}$ whose disease is not adequately controlled with topical therapies, or when those therapies are not advisable. In September 2017, it was also approved in Europe by EMA as a systemic first-line treatment for adults suffering from AD [9]. Dupilumab is supplied in a singledose pre-filled syringe and is administered by subcutaneous injection into the thigh, abdomen, or upper arm. The recommended dosage is a loading dose of $600 \mathrm{mg}$ (two 300-mg injections at different sites) followed by $300 \mathrm{mg}$ every other week, with rotation of the injection site. Dupilumab can be used with or without concomitant topical corticosteroids (TCS) [15]. It has also showed efficacy in patients with persistent asthma and elevated eosinophils, where it improved lung function, reduced exacerbations, and decreased Th2 biomarkers [16]. Randomized, double-blind, placebo-controlled trials involving adults who had moderate-to-severe $\mathrm{AD}$ were performed to check the applicability of dupilumab in the treatment of adults with moderate-to-severe AD. The first two clinical trials, SOLO 1 (NCT02277743) and SOLO 2 (NCT02277769), investigated the safety and efficacy of the dupilumab dosage regimen compared with placebo [17]. Patients were randomly assigned in a 1:1:1 ratio to receive weekly (qw) subcutaneous injections of dupilumab (300 mg) or placebo or the same dose of dupilumab every other week (q2w) alternating with placebo for 16 weeks. Significantly, more of the patients receiving dupilumab showed an improvement of at least $75 \%$ in the Eczema Area and Severity Index (EASI) score compared to the placebo group. The percentages of the patients who reached EASI-50 and EASI-90 as well as the improvement in the Scoring Atopic Dermatitis (SCORAD) and the decrease in the affected body surface area were all significantly higher in the dupilumab groups than in the placebo group (Table 1). A further phase III trial, LIBERTY AD CHRONOS, aimed to analyze the long-term management of moderate-to-severe $\mathrm{AD}$ with dupilumab and concomitant topical corticosteroids [18] (Table 1). At week 52, more of the patients who received dupilumab plus topical corticosteroids achieved the co-primary endpoints of IGA 0/1 and EASI-75 compared to the other two groups (Table 1). The efficacy of dupilumab has been demonstrated in adult patients with a history of inadequate response to/intolerance of CsA [19]. In the LIBERTY AD CAFÉ trial, 390 patients with an inadequate response to/intolerance of CsA or for whom CsA treatment was medically inadvisable were screened [19]. The proportion of the patients achieving EASI-75 at week 16 was significantly higher in the dupilumab qw + TCS and q2w + TCS groups compared to placebo + TCS. In all of the clinical studies performed in this field to date, dupilumab has shown a favorable safety profile with no dose-limiting toxicity and few adverse effects. The most common adverse events (AEs) associated with dupilumab are injection-site reactions, which mainly consist of transient erythema or edema. Conjunctivitis seems the only specific side effect, and this can be treated successfully with fluorometholone $0.1 \%$ eye drops or tacrolimus $0.03 \%$ eye ointment [20]. The reason why dupilumab causes conjunctivitis is not fully understood, and is currently being evaluated in ophthalmological subtrials. It was hypothesized that blockage of IL-4 and IL-13 would increase the activities of specific ligands involved in atopic keratoconjunctivitis, such as the OX40 ligand [21]. Generally, the severity of dupilumab-induced conjunctivitis in the majority (>90\%) of patients is only mild or moderate, and it resolves easily. Actually, there is only one case of bilateral conjunctivitis with consequent cicatricial ectropion associated with dupilumab therapy for $\mathrm{AD}$ in the literature [22]. 


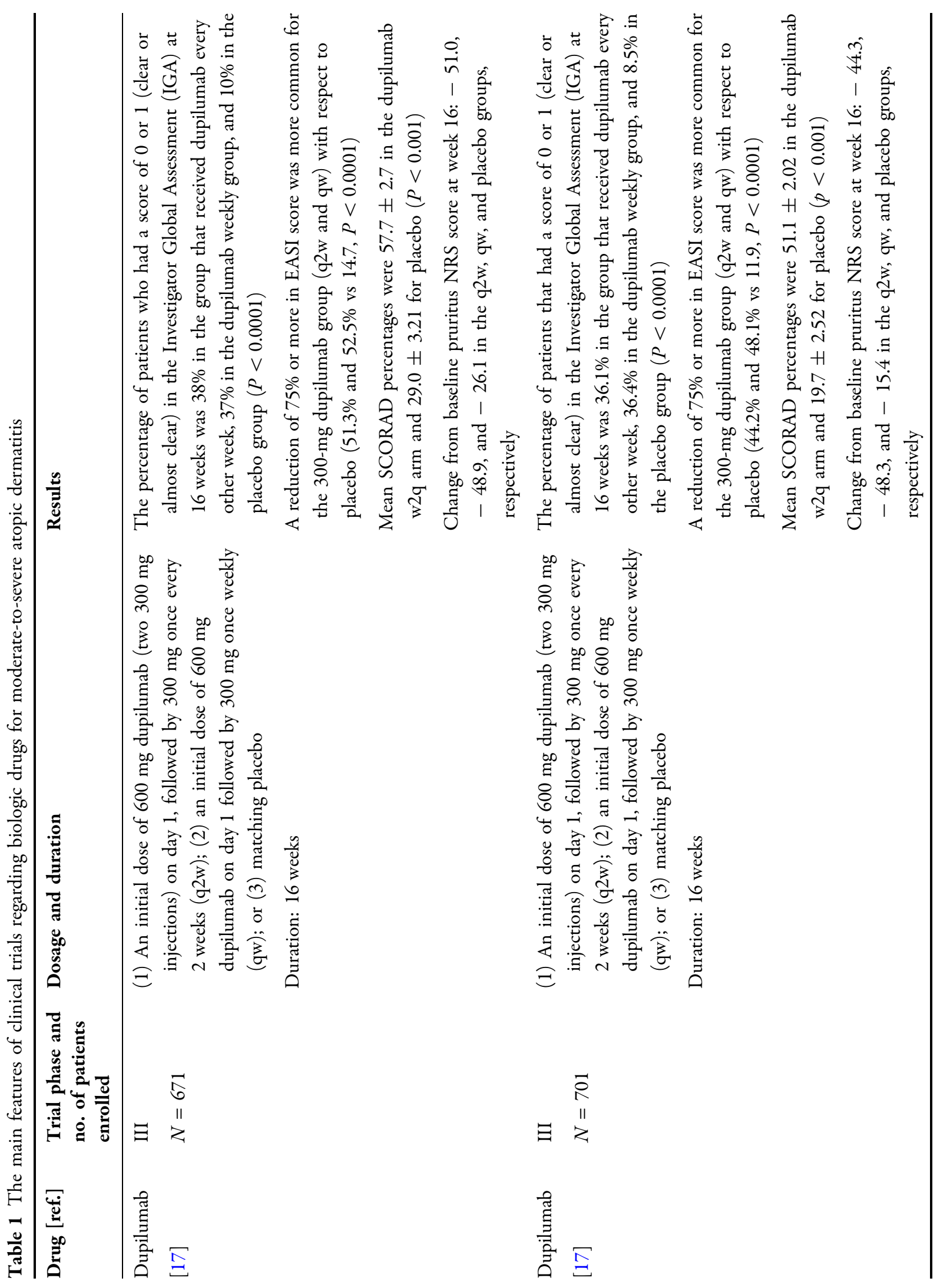




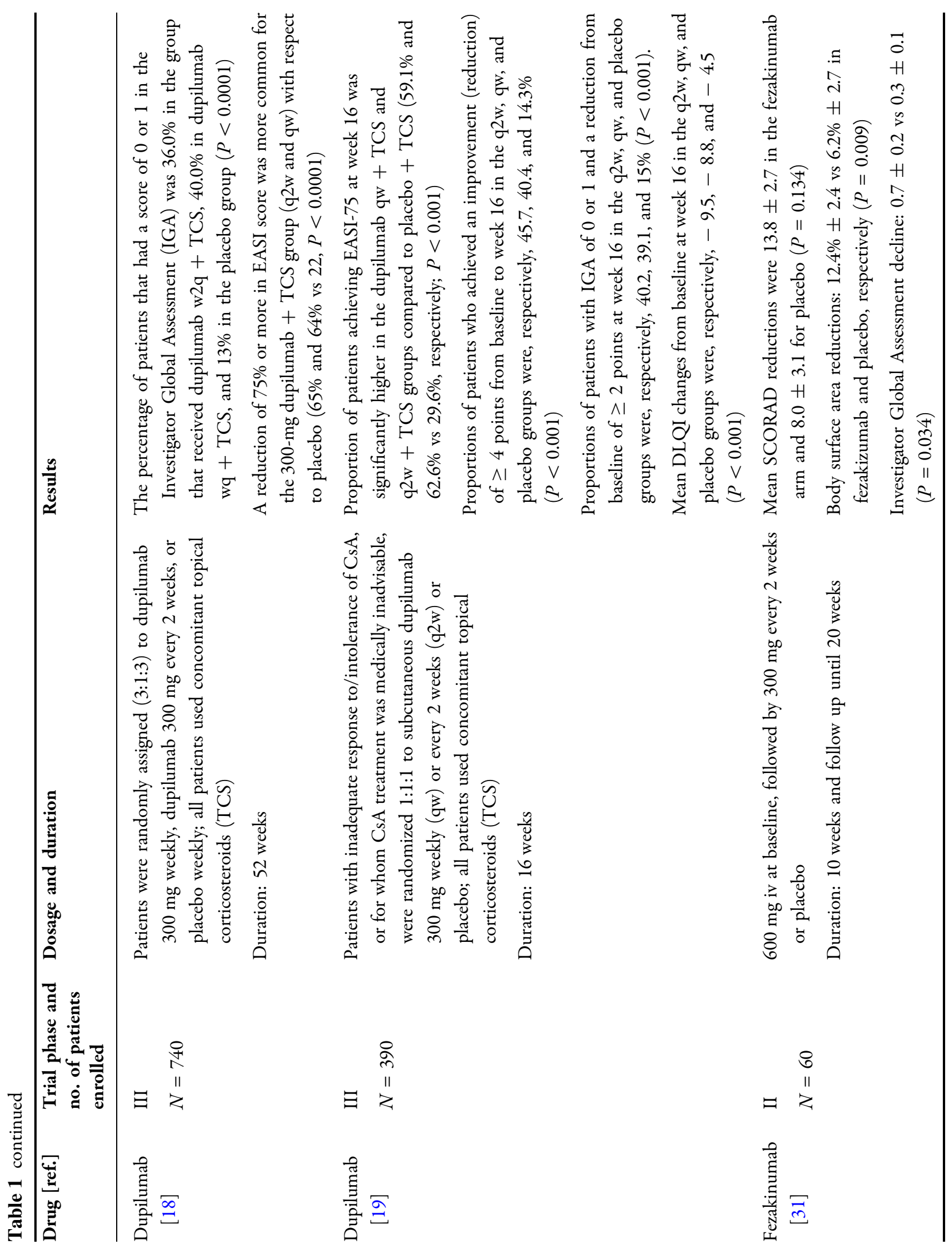




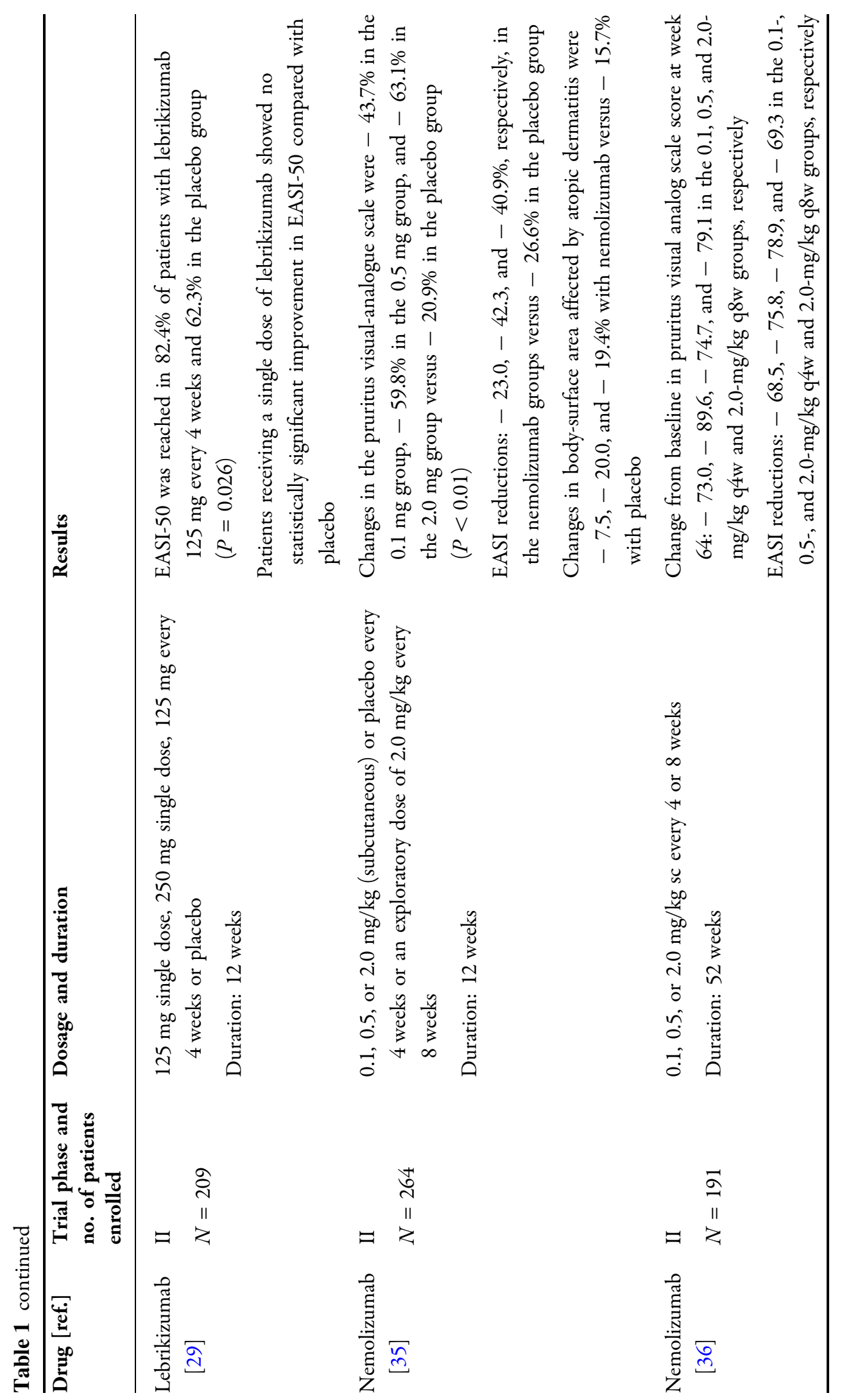




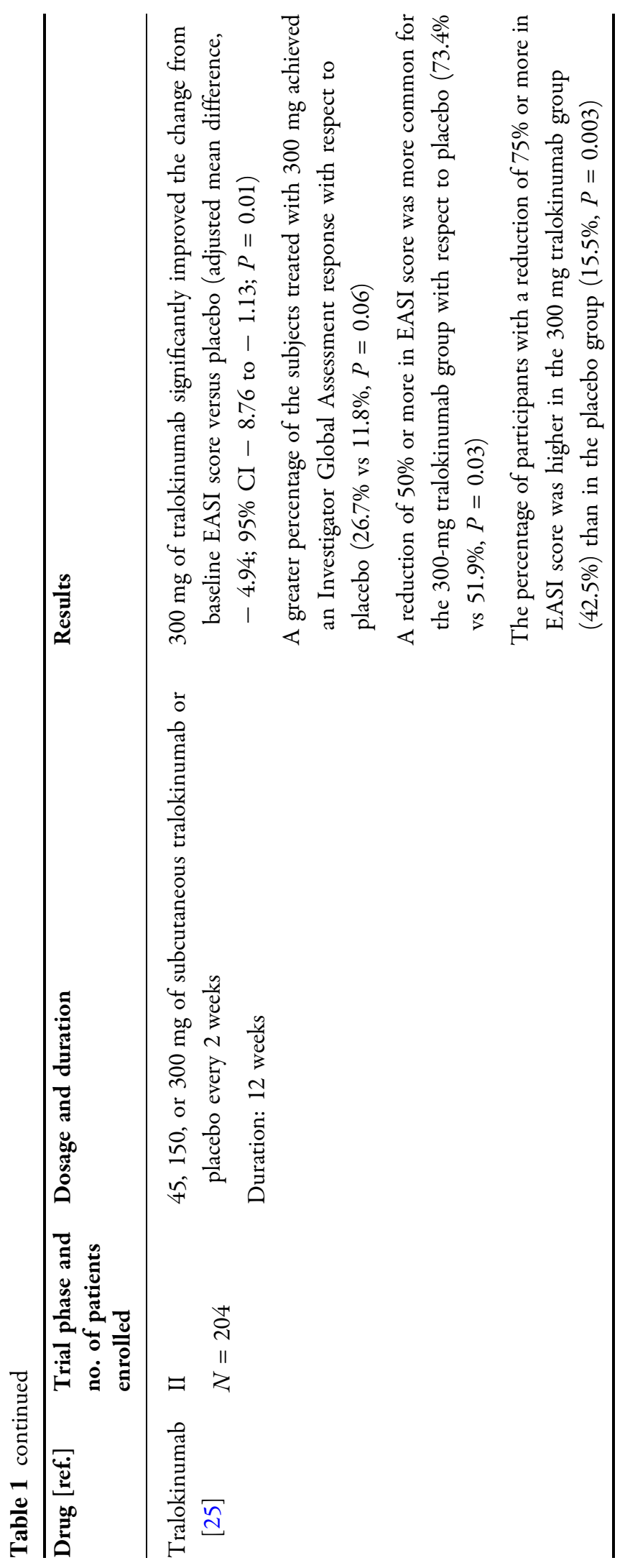


As with all therapeutic proteins, dupilumab has the potential for immunogenicity. Antidrug antibodies were developed by approximately $7 \%$ of patients with $\mathrm{AD}$ who received dupilumab $300 \mathrm{mg}$ every 2 weeks for 16 weeks in SOLO 1 and 2 [23]. Approximately 30\% of patients with anti-drug antibodies ( $2 \%$ overall) had neutralizing antibodies. Similarly, approximately $7 \%$ of the patients who received dupilumab $300 \mathrm{mg}$ once every 2 weeks (plus TCS) for 52 weeks in CHRONOS developed antibodies to dupilumab, with approximately $14 \%$ of those patients $(1 \%$ overall $)$ having neutralizing antibodies.

Several ongoing studies involving both children and adolescents will show if these subgroups of the $\mathrm{AD}$ population experience equally positive effects, expanding the treatment indication for dupilumab even further [24].

Tralokinumab is a human monoclonal antibody $(\mathrm{mAb})$ that specifically neutralizes IL-13 [25]. IL-13 is a pleiotropic Th2 cytokine that has been implicated in the pathogenesis of $\mathrm{AD}$, as it impairs skin barrier function by downregulating filaggrin [26, 27]. IL-13 is increased in both lesional and nonlesional skin of $\mathrm{AD}$ subjects, and correlates with disease severity [28]. A randomized, placebo-controlled, double-blind, clinical phase II study involving 204 adult patients with moderate-to-severe $\mathrm{AD}$ was performed [25]. At week 12, a dose of $300 \mathrm{mg}$ of tralokinumab every 2 weeks resulted in a significant improvement in $\mathrm{AD}$ compared with placebo (Table 1). Indeed, the drug significantly improved the EASI change from baseline versus placebo $(P=0.01$; Table 1$)$. In particular, a greater percentage of the $300 \mathrm{mg}$ tralokinumabtreated participants achieved EASI-50 versus placebo at week $12(73.4 \%$ vs $51.9 \%, P=0.03)$ as well as EASI-75 (42.5\% vs $15.5 \%, P=0.003)$, although concomitant TCS use may constitute a confounding factor that could explain the high placebo efficacy. Interestingly, patients with higher concentrations of biomarkers of increased IL-13 levels showed a better response to tralokinumab. The most frequent AEs in all groups were upper respiratory tract infections, nasopharyngitis, and headache [25]. All of these data indicated that tralokinumab treatment was associated with early improvements in $\mathrm{AD}$ symptoms and an acceptable safety and tolerability profile.

Lebrikizumab is a humanized monoclonal antibody that specifically targets IL-13 [29]. A phase II randomized, double-blind, prospective, placebo-controlled, multicenter study has been performed in adult subjects with moderate-tosevere $\mathrm{AD}$ [30]. In total, 209 patients were randomized (1:1:1:1) to lebrikizumab $125 \mathrm{mg}$ single dose, lebrikizumab $250 \mathrm{mg}$ single dose, lebrikizumab $125 \mathrm{mg}$ every 4 weeks for 12 weeks, or placebo every 4 weeks for 12 weeks. At week 12, EASI-50 was more commonly achieved in patients receiving lebrikizumab $125 \mathrm{mg}$ every 4 weeks than in placebo patients every 4 weeks ( $82.4 \%$ vs $62.3 \%, P=0.026)$. The high placebo performance may due to the background of daily TCS application (Table 1). Lebrikizumab was well tolerated; adverse events were similar between groups $(66.7 \%$ all lebrikizumab vs $66.0 \%$ placebo) and were mostly mild or moderate; no AE dose-response relationships were found for the drug. In that study, the efficacy of lebrikizumab $125 \mathrm{mg}$ every 4 weeks led to a significant improvement in patients with moderate-to-severe AD.

\section{IL-22 Inhibitor}

IL-22 was found to be increased in both acute and chronic $\mathrm{AD}$ lesions, correlating with $\mathrm{AD}$ severity [28]. It is produced primarily by Th22 cells and is able to impair epithelial barrier function through its effect on keratinocytes [31].

Fezakinumab is a fully human monoclonal antibody directed against IL-22. A recent randomized, double-blind, placebo-controlled trial with intravenous fezakinumab monotherapy every 2 weeks for 10 weeks and follow-up assessments until 20 weeks has been completed [32]. Sixty-seven adult AD patients were assessed for eligibility, and 60 patients were randomized 2:1 to fezakinumab $(n=40)$ or placebo $(n=20)$. Results showed that the SCORAD improvement was significantly greater with fezakinumab versus placebo in patients with severe $\mathrm{AD}$ at 12 weeks $(36.4 \%$ vs $22.3 \%$, $P<0.05)$ and 20 weeks $(46.2 \%$ vs $22.6 \%$, 
$P<0.01$ ), while patients with moderate AD seemed to be less responsive to treatment (Table 1) [31]. AEs occurred with similar frequencies in the fezakinumab and placebo groups [32]. Taken together, these data showed that fezakinumab was well tolerated, with sustained clinical improvements after the last drug dosing.

\section{IL-31 Receptor Inhibitor}

It is already known that the skin expression of IL-31 and IL-31R is increased in patients with AD [33]. Moreover, AD subjects also show higher serum levels of IL-31 as well as IL-31producing lymphocytes [34]. In addition, both serum and skin IL-31 levels have been shown to correlate with $\mathrm{AD}$ severity $[33,34]$, indicating an important role for IL-31 in AD pathogenesis apart from itch pathophysiology [35].

Nemolizumab is a monoclonal antibody directed against IL-31 receptor A [33]. A phase II, randomized, double-blind, placebo-controlled, 12-week trial involving 264 adult patients with moderate-to-severe $\mathrm{AD}$ has been recently completed [36]. Patients received subcutaneous nemolizumab (at doses of $0.1,0.5$, or $2.0 \mathrm{mg} / \mathrm{kg}$ of body weight) or placebo every 4 weeks. Significant reductions in pruritus were seen from baseline to week 12 in all arms receiving nemolizumab every 4 weeks; the result was statistically significant with respect to placebo in all drug groups (Table 1). EASI score changes were significantly higher for 0.5 and $2 \mathrm{mg}$ nemolizumab with respect to placebo (Table 1). AD exacerbation, nasopharyngitis, upper respiratory tract infection, peripheral edema, and increased creatine kinase levels were the most common AEs registered. In particular, exacerbation of $\mathrm{AD}$ and peripheral edema were more common in the nemolizumab groups than in the placebo group.

More recently, the long-term efficacy and safety of nemolizumab injected subcutaneously every 4 weeks $(\mathrm{q} 4 \mathrm{w})$ or 8 weeks $(\mathrm{q} 8 \mathrm{w})$ were evaluated in a phase II, 52-week, double-blind extension trial [37]. Patients $(n=191)$ continued the previous nemolizumab dose $(0.1,0.5$, or $2.0 \mathrm{mg} / \mathrm{kg}$ q4w or $2.0 \mathrm{mg} / \mathrm{kg} \mathrm{q} 8 \mathrm{w}$ ) [36]. An improvement from baseline in the pruritus visual analog scale score was maintained/increased from weeks 12 to 64 , with the greatest improvement seen for the $0.5-\mathrm{mg} / \mathrm{kg} \mathrm{q} 4 \mathrm{w}$ group (Table 1). The EASI improvement was also maintained/increased to week 64, with the highest results observed with a dosage of $2.0 \mathrm{mg} / \mathrm{kg} \mathrm{q} 4 \mathrm{w}$ (Table 1), showing that nemolizumab for up to 64 weeks was efficacious and well tolerated overall in patients with moderateto-severe $\mathrm{AD}$ inadequately controlled by topical therapy. The most common AEs in patients treated over the long term with nemolizumab ( $\geq 5 \%$ of patients were randomized to nemolizumab throughout the study period) were nasopharyngitis (27\%), exacerbation of $\mathrm{AD}$ (25\%), increased blood creatine phosphokinase (11\%), upper respiratory tract infection (9\%), headache $(8 \%)$, peripheral edema $(6 \%)$, and impetigo (6\%).

\section{CONCLUSION}

The frequency of $\mathrm{AD}$ is increasing all over the world, especially in industrialized countries and urbanized areas. The frequency of this disease in adults also seems to be increasing, although it is probably underdiagnosed. Due to its chronicity and subjective symptoms, the disease greatly impacts on the quality of life of the patients. Currently, only few systemic immunosuppressive drugs are available for moderate-to-severe forms, and CsA is the only licensed drug for the treatment. Moreover, the important side effects of immune suppressors limit their use. Therefore, having more secure and effective medicines would be desirable. Over the last few years, increased knowledge about $\mathrm{AD}$ pathogenesis has made it possible to develop new therapeutic agents capable of blocking individual mediators of inflammation. Some monoclonal antibodies against specific cytokines (IL-4, IL-13, IL-22, IL31) have been developed. Data obtained from the trials regarding efficacy and safety are encouraging for most of the agents. Dupilumab (anti-IL-4/IL-13) is the only biologic agent approved for $\mathrm{AD}$ in some countries. In conclusion, biologic therapies will probably markedly change the natural history of AD. Indeed, they 
will hopefully be able to increase the diseasefree periods and decrease the number of exacerbations, thus improving the quality of life of AD patients.

\section{ACKNOWLEDGEMENTS}

Funding. No funding or sponsorship was received for this study or the publication of this article.

Authorship. All named authors meet the International Committee of Medical Journal Editors (ICMJE) criteria for authorship for this manuscript, take responsibility for the integrity of the work as a whole, and have given final approval for the version to be published.

Disclosures. Cataldo Patruno is an investigator for AbbVie and Almirall, an advisory board member and speaker for Sanofi Genzyme, and a speaker for Novartis. Gabriella Fabbrocini, Maddalena Napolitano, Matteo Megna, and Nicola Balato have nothing to disclose.

Compliance with Ethics Guidelines. This article is based on previously conducted studies and does not involve any new study of human or animal subjects performed by any of the authors.

Open Access. This article is distributed under the terms of the Creative Commons Attribution-NonCommercial 4.0 International License (http://creativecommons.org/licenses/ by-nc/4.0/), which permits any noncommercial use, distribution, and reproduction in any medium, provided you give appropriate credit to the original author(s) and the source, provide a link to the Creative Commons license, and indicate if changes were made.

\section{REFERENCES}

1. Megna M, Napolitano M, Patruno C, Villani A, Balato A, Monfrecola G, Ayala F, Balato N. Systemic treatment of adult atopic dermatitis: a review. Dermatol Ther (Heidelb). 2017;7:1-23.

2. Napolitano M, Megna M, Patruno C, Gisondi P, Ayala F, Balato N. Adult atopic dermatitis: a review. G Ital Dermatol Venereol. 2016;151:403-11.

3. Dinulos JG, Trickett A, Crudele C. New science and treatment paradigms for atopic dermatitis. Curr Opin Pediatr. 2018;30:161-8.

4. Katsarou A, Armenaka MC. Atopic dermatitis in older patients: particular points. J Eur Acad Dermatol Venereol. 2011;25:12-8.

5. Silvestre Salvador JF, Romero-Pérez D, EncaboDurán B. Atopic dermatitis in adults: a diagnostic challenge. J Investig Allergol Clin Immunol. 2017;27:78-88.

6. Kim DH, Li K, Seo SJ, et al. Quality of life and disease severity are correlated in patients with atopic dermatitis. J Korean Med Sci. 2012;27:1327-32.

7. Megna M, Patruno C, Balato A, Rongioletti F, Stingeni L, Balato N. An Italian multicentre study on adult atopic dermatitis: persistent versus adult-onset disease. Arch Dermatol Res. 2017;309:443-52.

8. Calzavara Pinton P, Cristaudo A, Foti C, et al. Diagnosis and management of moderate to severe adult atopic dermatitis: a consensus by the Italian Society of Dermatology and Venereology (SIDeMaST), the Italian Association of Hospital Dermatologists (ADOI), the Italian Society of Allergy, Asthma and Clinical Immunology (SIAAIC), and the Italian Society of Allergological, Environmental and Occupational Dermatology (SIDAPA). G Ital Dermatol Venereol. 2018;153:133-45.

9. Wollenberg A, Barbarot S, Bieber T, et al. Consensus-based European guidelines for treatment of atopic eczema (atopic dermatitis) in adults and children: part II. J Eur Acad Dermatol Venereol. 2018;32:850-78.

10. Rerknimitr P, Otsuka A, Nakashima C, Kabashima $K$. The etiopathogenesis of atopic dermatitis: barrier disruption, immunological deragement, and pruritus. Inflamm Regen. 2017;37:14.

11. Eshtiaghi P, Gooderham MJ. Dupilumab: an evidence-based review of its potential in the treatment of atopic dermatitis. Core Evid. 2018;13:13-20.

12. Schmitt J. After the approval of dupilumab for moderate-to-severe atopic dermatitis: what is next on the research agenda? $\mathrm{Br} \mathrm{J}$ Dermatol. 2018;178:992-3. 
13. Seegräber M, Srour J, Walter A, Knop M, Wollenberg A. Dupilumab for treatment of atopic dermatitis. Expert Rev Clin Pharmacol. 2018;11:467-74.

14. Bao L, Zhang H, Chan LS. The involvement of the JAK-STAT signaling pathway in chronic inflammatory skin disease atopic dermatitis. Jakstat. 2013;2:e24137.

15. US FDA. Dupixent (dupilumab) injection, for subcutaneous use: US prescribing information. 2017. https://www.fda.gov. Accessed 2 May 2018.

16. Wenzel S, Ford L, Pearlman D, et al. Dupilumab in persistent asthma with elevated eosinophil levels. N Engl J Med. 2013;368:2455-66.

17. Simpson EL, Bieber T, Guttman-Yassky E, et al. Two phase 3 trials of dupilumab versus placebo in atopic dermatitis. N Engl J Med. 2016;375:2335-48.

18. Blauvelt A, de Bruin-Weller M, Gooderham M, et al. Long-term management of moderate-to-severe atopic dermatitis with dupilumab and concomitant topical corticosteroids (LIBERTY AD CHRONOS): a 1-year, randomised, double-blinded, placebo-controlled, phase 3 trial. Lancet. 2017;389:2287-303.

19. de Bruin-Weller $\mathrm{M}$, Thaçi D, Smith $\mathrm{CH}$, et al. Dupilumab with concomitant topical corticosteroid treatment in adults with atopic dermatitis with an inadequate response or intolerance to ciclosporin $\mathrm{A}$ or when this treatment is medically inadvisable: a placebo-controlled, randomized phase III clinical trial (LIBERTY AD CAFÉ). Br J Dermatol. 2018;178:1083-101.

20. Wollenberg A, Ariens L, Thurau S, van Luijk C, Seegräber $M$, de Bruin-Weller $M$. Conjunctivitis occurring in atopic dermatitis patients treated with dupilumab-clinical characteristics and treatment. J Allergy Clin Immunol Pract. 2018;18:30089-8 (pii: S2213-2198).

21. Mennini M, Dahdah L, Fiocchi A. Two phase 3 trials of dupilumab versus placebo in atopic dermatitis. N Engl J Med. 2017;376:1090.

22. Barnes AC, Blandford AD, Perry JD. Cicatricial ectropion in a patient treated with dupilumab. Am J Ophthalmol Case Rep. 2017;7:120-2.

23. Shirley M. Dupilumab: first global approval. Drugs. 2017;77:1115-21.

24. Blakely K, Gooderham M, Papp K, Dupilumab A. Monoclonal antibody for atopic dermatitis: a review of current literature. Skin Therapy Lett. 2016;21:1-5.

25. Wollenberg A, Howell MD, Guttman-Yassky E, Silverberg JI, Kell C, Ranade K, Moate R, van der
Merwe R. Treatment of atopic dermatitis with tralokinumab, an anti-IL-13 monoclonal antibody. J Allergy Clin Immunol. 2018;18:30850-9. https:// doi.org/10.1016/j.jaci.2018.05.029 (pii: S00916749).

26. Hamann CR, Thyssen JP. Monoclonal antibodies against interleukin 13 and interleukin 31RA in development for atopic dermatitis. J Am Acad Dermatol. 2018;78:S37-42.

27. Kim BE, Leung DY, Boguniewicz M, et al. Loricrin and involucrin expression is down-regulated by Th2 cytokines through STAT-6. Clin Immunol. 2008;126:332-7.

28. Ungar B, Garcet S, Gonzalez J, Dhingra N, da Rosa JC, Shemer A, et al. An integrated model of atopic dermatitis biomarkers highlights the systemic nature of the disease. J Invest Dermatol. 2017;137:603-13.

29. Tazawa T, Sugiura H, Sugiura Y, Uehara M. Relative importance of IL-4 and IL-13 in lesional skin of atopic dermatitis. Arch Dermatol Res. 2004;295:459-64.

30. Simpson EL, Flohr C, Eichenfield LF, et al. Efficacy and safety of lebrikizumab (an anti-IL-13 monoclonal antibody) in adults with moderate-to-severe atopic dermatitis inadequately controlled by topical corticosteroids: a randomized, placebo-controlled phase II trial (TREBLE). J Am Acad Dermatol. 2018;78(863-871):e11.

31. Gittler JK, Shemer A, Suarez-Farinas M, et al. Progressive activation of $\mathrm{T}(\mathrm{H}) 2 / \mathrm{T}(\mathrm{H}) 22$ cytokines and selective epidermal proteins characterizes acute and chronic atopic dermatitis. J Allergy Clin Immunol. 2012;130:1344-54.

32. Guttman-Yassky E, Brunner PM, Neumann AU, et al. Efficacy and safety of fezakinumab (an IL22 monoclonal antibody) in adults with moderateto-severe atopic dermatitis inadequately controlled by conventional treatments: a randomized, doubleblind, phase $2 \mathrm{a}$ trial. J Am Acad Dermatol. 2018;78:872-81.

33. Hamann CR, Thyssen JP. Monoclonal antibodies against interleukin 13 and interleukin 31RA in development for atopic dermatitis. J Am Acad Dermatol. 2018;78:S37-42.

34. Szegedi K, Kremer AE, Kezic S, et al. Increased frequencies of IL-31-producing $\mathrm{T}$ cells are found in chronic atopic dermatitis skin. Exp Dermatol. 2012;21:431-6.

35. Sonkoly E, Muller A, Lauerma AI, et al. IL-31: a new link between $\mathrm{T}$ cells and pruritus in atopic skin 
inflammation. J Allergy Clin Immunol. 2006;117:411-7.

36. Ruzicka T, Hanifin JM, Furue M, et al. Anti-Interleukin-31 receptor A antibody for atopic dermatitis. N Engl J Med. 2017;376:826-35.
37. Kabashima K, Furue M, Hanifin JM, Pulka G, Wollenberg A, Galus R, Etoh T, Mihara R, Nakano M, Ruzicka T. Nemolizumab in patients with moderate-to-severe atopic dermatitis: randomized, phase II, long-term extension study. J Allergy Clin Immunol. 2018;10(18):30698-5 pii: S0091-6749. 